\title{
Forecasting Directional Changes in the FX Markets
}

\author{
Amer Bakhach, Edward P. K. Tsang, Hamid Jalalian \\ Centre for Computational Finance and Economic Agents (CCFEA) \\ University of Essex \\ Colchester, UK \\ abakhaa, Edward, hamidj(@essex.ac.uk)
}

\begin{abstract}
Most of existing studies sample markets' prices as time series when developing models to predict market's trend. Directional Changes (DC) is an approach to summarize market prices other than time series. DC marks the market as downtrend or uptrend based on the magnitude of prices changes. In this paper we address the problem of forecasting trend's direction in the foreign exchange (FX) market under the DC framework. In particularly we aim to answer the question of whether the current trend will continue for a specific percentage before the trend ends. We propose one single independent variable to make the forecast. We assess the accuracy of our approach using three currency pairs in the FX market; namely EUR/CHF, GBP/CHF, and USD/JPY. The experimental results show that the accuracy of the proposed forecasting model is very good; in some cases, forecasting accuracy was over $80 \%$. However, under particular settings the accuracy may not outperform dummy prediction. The results confirm that directional changes are predictable, and the identified independent variable is useful for forecasting under the DC framework.
\end{abstract}

Keywords - FX rates, financial forecasting, directional changes.

\section{INTRODUCTION}

Trading in the FX markets averaged $\$ 5.1$ trillion per day in April 2016. This is down from 5.3 trillion in April 2013; and up from 4.0 trillion in April 2010 [1]. Therefore the prediction of exchange rates has been an attractive objective for many studies. For instance, the authors in [2] introduce an Echo State Networks model in order to forecast the dynamic of several currency pairs. The authors in [3] present a model to evaluate the efficacy of Support Vector Regression to predict EUR/USD exchange rate. To this end, they use several variables, those derived from the Bollinger Bands indicator, as explanatory variables [3]. In [4], the authors use an Intelligent Exchange Rates Prediction System based on cloud computing to build a linear regression model with the aim of predicting the dynamic of currency exchange rates. The study reported in [5] compares the ability of Artificial Neural Network with different ARCH and GARCH models to predict the daily exchange rate of EUR/USD. A model that combine Neural Network with ARIMA is used to predict two currency pair's rates in [6].

The literature also encompasses several studies those aim to forecast the direction of market's trend. For example, the authors in [7] propose a Hidden Markov Model to predict next days' price change direction. A Bayesian multilayer perceptron model is proposed in [8] to predict the direction of daily closed value of the 'All Ords' Australian financial index. Other studies refer to the same problem as 'forecasting turning points'. For instance, the authors in [9] propose two models based on fuzzy logic and neural networks and applied them to predict turning point in the S\&P500 index. As a general note, most studies in the literature use time series to develop and test their models. In other words, these studies consider market prices sampled at fixed time intervals, be that days, minutes, etc.

Directional Change (DC) is a different approach to summarize prices movement [10]. Under the DC framework the market is simplified as alternating uptrend and downtrend. A trend is identified as a change in market price larger than, or equal to, a specific threshold. This threshold is set by the observer. A trend ends whenever a price change of the same threshold is observed in the inverse direction. For example, a market downtrend ends when we observe a price rise equal to the selected threshold; in this case we say that the market changes its direction to an uptrend. Similarly a market uptrend ends when we observe a price drop equal to the same threshold. Recently, many studies have shown that the DC framework is helpful in studying the FX markets [11-17]. However, the problem of forecasting trend's direction, or turning point, has not been considered from the DC perspective yet.

In this paper we consider the market trends' forecasting problem based on the DC context. In this paper we are primarily interested in forecasting the magnitude of price change. In contrast, most of existing approaches (e.g. [7-9]) do not take into concern the magnitude of price's change. They aim to answer the question: "will today's close price extend yesterday's trend?" In this paper, the task is to predict whether the current uptrend, or downtrend, will continue in the same direction for a specific percentage before the trend ends. Answering this question could help a trader to decide whether to take a long or short position. To this end, we introduce one independent variable. We provide several experiments to show that our approach is useful for the proposed forecasting problem in the FX market.

This paper continues as follow: Directional Change is explained in Section II. Section III provides the formal definition of the proposed forecasting problem. In Section IV we present our approach to solve the introduced forecasting problem. Section V provides the details of our experiments and the testing methodology. The experimental results are reported and discussed in Section VI. We conclude in Section VII.

\section{DiRectional Changes: AN OVERVIEW}

Directional change (DC) is an approach to summarize price changes other than time series [10]. In this section, we explain how market prices are sampled based on the DC concept. Under the DC framework, the market is summarized into alternating uptrends and downtrends. Let us consider a market in a downtrend. Let $P_{E X T}$ be the lowest price in this downtrend and $P_{c}$ be the current price. We say that the market switches its direction from downtrend to uptrend whenever $P_{c}$ becomes 


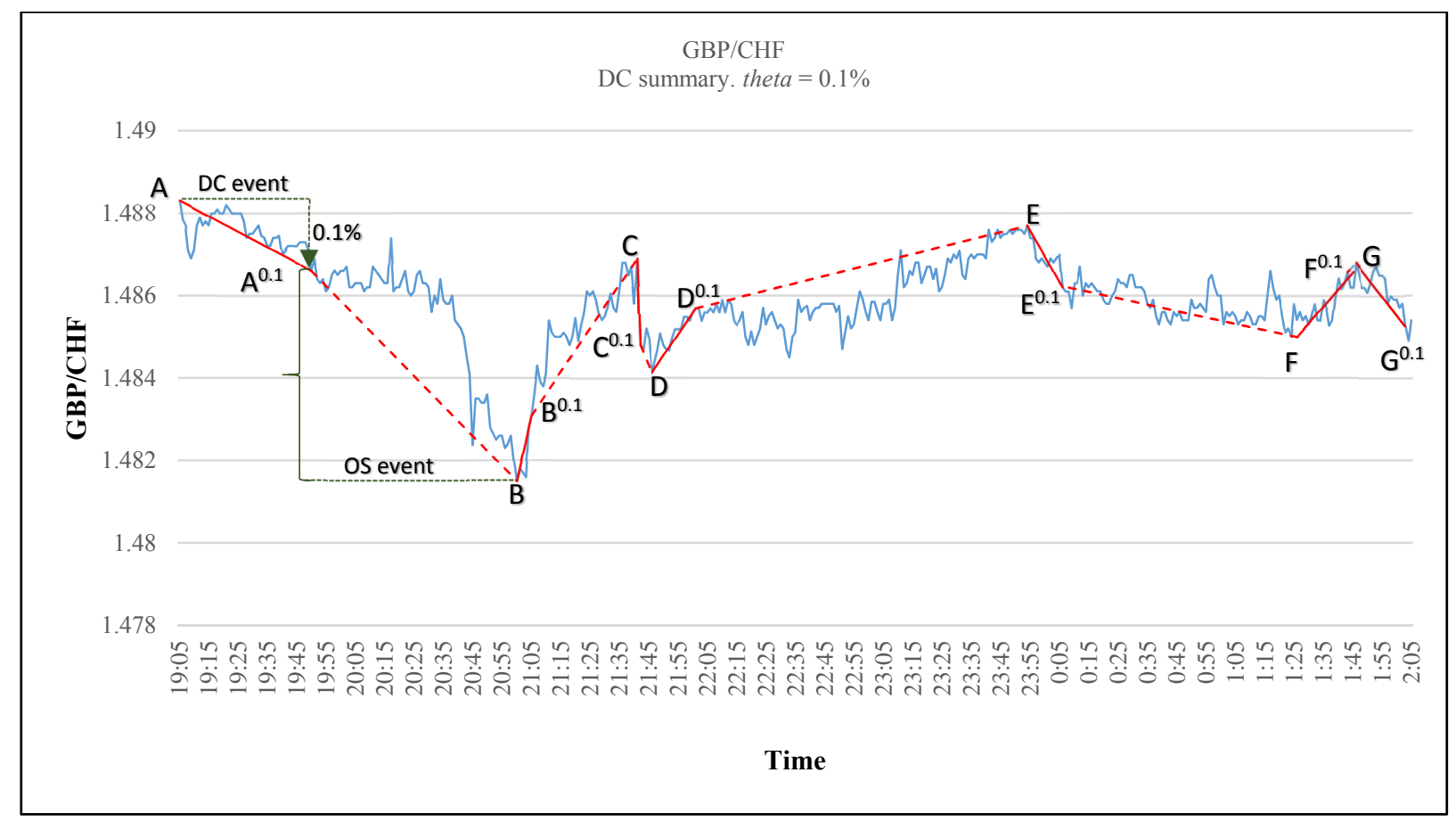

Fig. 1. An example of a DC-based summary. The blue line indicates GBP/CHF mid-prices sampled minute by minute from 1/1/2013 19:05 to 1/2/2013 02:05 GMT. theta $=0.1 \%$. Solid red lines represent DC events. Dashed red lines represent OS events. Each of the points A, B, C..etc, represents a specific time-minute.

greater than $P_{E X T}$ by at least theta (where theta is the threshold predetermined by the observer; usually expressed as percentage). Similarly, if the market is in uptrend, $P_{E X T}$ would refer to the highest price in this uptrend. We say that the market switches its direction from an uptrend to a downtrend if $P_{c}$ is lower than $P_{E X T}$ by at least theta. Each trend comprises a DC event and an overshoot (OS) event (see Fig. 1). Formally, a DC event is detected when we come across a price $P_{c}$ that satisfies (1):

$$
\left|\frac{P_{c}-P_{E X T}}{P_{E X T}}\right| \geq \text { theta }
$$

If (1) holds, then the time at which the market traded at $P_{E X T}$ is called an 'extreme point' (e.g. points A and D in Fig. 1), and the time at which the market trades at $P_{c}$ is called a DC confirmation point, or DCC point for short (e.g. points $\mathrm{A}^{0.1}$ and $\mathrm{D}^{0.1}$ in Fig. 1). Note that whilst an extreme point is the end of one trend, it is also the start of the next trend, which has an opposite direction. An extreme point is only recognized in hindsight; precisely at the DCC point. For example, in Fig. 1, at point $\mathrm{A}^{0.1}$ we confirm that point $\mathrm{A}$ is an extreme point. Similarly, in Fig. 1, at point $\mathrm{D}^{0.1}$ we confirm that point $\mathrm{D}$ is an extreme point. A DC event starts with an extreme point and ends with a DCC point. An (OS) event starts at the DCC point and ends at the next extreme point.

The DC summary of a given market is the identification of the DC and OS events, governed by the threshold theta. Fig. 1 shows an example of a DC summary. Note that for a given time series and a predetermined threshold, the DC summary is unique. However, we may generate multiple DC summaries for the same considered prices series by selecting multiple thresholds. The chosen threshold determines what constitutes a directional change. Had a greater threshold been chosen, less directional changes would have been concluded between the points in Fig. 1 (see Fig. 2 for example). We refer to a specific DC event by its starting point, i.e. extreme point, and its DCC point. For example, in Fig. 1 the DC event which starts at point $\mathrm{B}$ and ends at point $\mathrm{B}^{0.1}$ is denoted as $\left[\mathrm{BB}^{0.1}\right]$.

In traditional time series data is sampled based on fixed time interval (e.g. days, months). The major difference between time series and DC summary is that the latter focuses on the magnitude of price change and time is the varying element. For example, in Fig. 1 the DC event $\left[\mathrm{AA}^{0.1}\right]$ took 50 minutes; whereas the $\mathrm{DC}$ event $\left[\mathrm{EE}^{0.1}\right]$ took 12 minutes. Note that the price changes during both $\mathrm{DC}$ events, $\left[\mathrm{AA}^{0.1}\right]$ and $\left[\mathrm{EE}^{0.1}\right]$, are equal to the selected threshold $0.1 \%$. Under the DC framework, any price change less than the selected threshold is not considered as a trend. A more in-depth analysis of the differences between time series and DC analysis can be found in [11].

Many studies provide evidence that the DC framework is helpful in studying the FX markets. For example, [12] reveals twelve scaling laws, based on the DC concept, which uncover new facts in the FX market. The aim of these scaling laws is to establish mathematical relationships among price moves, duration and trend frequency. Reference [13] presents the socalled Scale of Market Quakes (SMQ) based on the DC concept. SMQ aims to quantify FX market activity during significant economic and political events declarations. For this purpose, SMQ measures the excess price moves during the OS event. Moreover, an example of how to utilize the DC concept to develop a High Frequency Trading model is presented in 


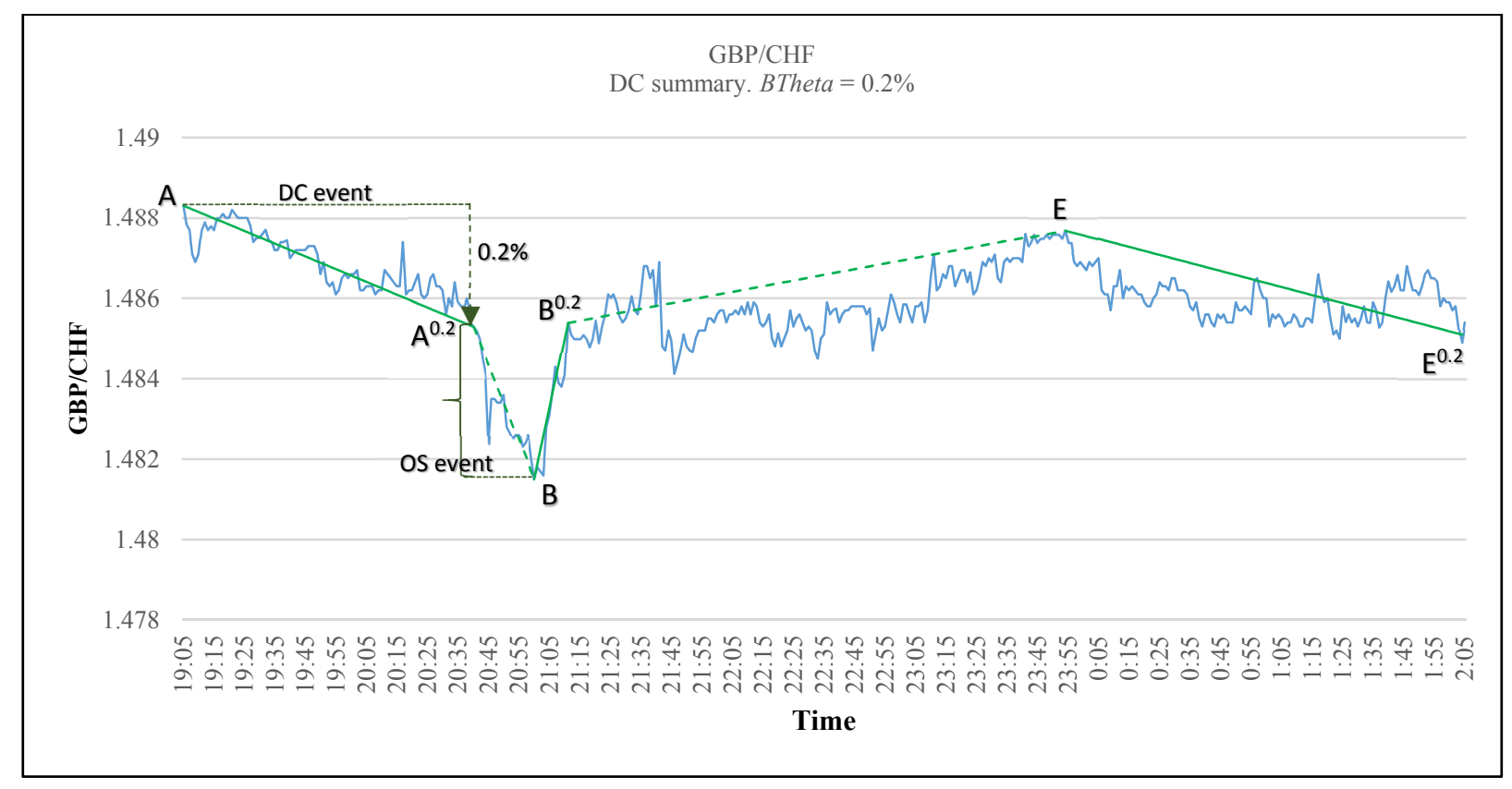

Fig. 2. A DC summary for GBP/CHF mid-prices sampled minute by minute from 1/1/2013 19:05 to 1/2/2013 02:05. BTheta $=0.2 \%$. Solid green lines represent DC events. Dashed green lines represent OS events.

[14]. Furthermore, a study that deciphers FX market activities based on the DC concept is reported in [15]. It explains how minor differences in market activities can change the market's trend under definite conditions. The authors in [16] propose a trading strategy using a combination of the DC framework and genetic programming. A recent study reported in [17] proposes a model to measure the liquidity in the FX market based on the DC framework. Their approach seeks to model market dynamic in order to predict stress in financial markets. They suggest that their approach can be used as an early warning system [17]. Finally, an approach to profiling companies and financial markets is introduced in [11]. The adopted methodology is based on the DC analysis of high frequency price movements. They conclude that information obtained through DC-based analysis and from time series complement each other [11]. The DC concept [10] is similar to the zigzag ${ }^{1}$ indicator used by traders. The major difference is that a trend, under the DC methodology, is fragmented into: 1) a DC event of fixed percentage, equal to the selected threshold, and 2) an OS event represented by the remaining part of the trend before it reverses. Such partitioning is not part of the zigzag indicator. Many studies provide evidence that such partitioning could be very useful to analyze the FX market (e.g. [12] [15]).

\section{PROBLEM Formulation}

In this section we propose a formulation of the problem of trend's direction prediction under the DC framework. This formulation is not related to any of the previously cited works. First, we shall introduce the concept of Big-Theta in Section A, and the dependent variable BBTheta in Section B. Then we shall formulate the forecasting problem in Section C.

\section{A. The Concept of Big-Theta}

Before formalizing our objective, we need to introduce the concept of Big-Theta. The concept of Big-Theta states that a DC event of threshold BTheta will embrace at least one DC event of a smaller threshold STheta (with BTheta $>$ STheta). As stated in Section II, for the same price series we may obtain several DC summaries by using multiple thresholds. For instance Fig. 2 illustrates a DC summary of the same GBP/CHF prices considered in Fig.1 using another threshold (BTheta $=$ $0.2 \%$ ). Eventually, the smaller the threshold is the more DC events we get. For example, in Fig. 1 (with threshold of $0.1 \%$ ) we observe four downtrends and three uptrends. Whereas in Fig. 2 (with threshold of $0.2 \%$ ) we observe only two downtrends and one uptrend. In general, the total number of trends of threshold BTheta is less than the total number of trends of threshold STheta (for BTheta $>$ STheta).

Let $E P^{0.002}$ be the set of all extreme points shown in Fig. 2 . $E P^{0.002}=\{\mathrm{A}, \mathrm{B}, \mathrm{E}\}$. Similarly, Let $E P^{0.001}$ be the set of all extreme points shown in Fig. 1.EP $P^{0.001}=\{\mathrm{A}, \mathrm{B}, \mathrm{C}, \mathrm{D}, \mathrm{E}, \mathrm{F}, \mathrm{G}\}$. An important remark is that each point in $E P^{0.002}$ is also a point of $E P^{0.001}$. In general, an extreme point of DC event of threshold $B$ Theta is also an extreme point for any other DC event of threshold STheta (with BTheta $>$ STheta). This statement can be proved logically: any DC event of threshold $0.20 \%$ implies, implicitly, a DC event of any threshold less than $0.20 \%$ (e.g. $0.10 \%, 0.15 \%, 0.05 \%)$. However, the inverse is not true. For instance, the extreme points $\mathrm{C}$ and $\mathrm{D}$ are elements of $E P^{0.001}$ but they are not elements of $E P^{0.002}$ (i.e. none of $\mathrm{C}$ and $\mathrm{D}$ is an extreme point of a DC event of threshold $0.2 \%$ ). By definition, the elements of $E P^{0.001}$, and $E P^{0.002}$ are sorted chronologically.

\footnotetext{
${ }^{1}$ http://www.investopedia.com/terms/z/zig_zag_indicator.asp
} 
For example, points $\mathrm{A}$ and $\mathrm{B}$ in $E P^{0.001}$ are the extreme points of the $1^{\text {st }}$ and $2^{\text {nd }}$ trends in Fig. 1 respectively.

\section{B. The Boolean Variable BBTheta}

In this section, we introduce a Boolean variable BBTheta. BBTheta is True if and only if an extreme point for a DC event under STheta is also an extreme point for a DC event under BTheta.

Fig. 3 shows the synchronization of the two DC summaries shown in Fig. 1 and Fig. 2. For each DC event of threshold STheta we associate a value of BBTheta. Let BBTheta ${ }^{i}$ be the value of $B B$ Theta associated to the $i^{\text {th }}$ DC event of the DC summary of threshold STheta. BBTheta can be only True or False. The value of BBTheta ${ }^{i}$ is defined as follows:

If the extreme point of the $i^{\text {th }}$ trend of the DC summary of threshold STheta is also an extreme point of another trend of another DC summary of threshold BTheta then BBTheta ${ }^{i}=$ True; otherwise BBTheta ${ }^{i}=$ False.

For example, in Fig. 3 point B is the extreme point of the $2^{\text {nd }}$ trend of threshold STheta $=0.1 \%$. The same point, B, is also the extreme point of another trend of threshold BTheta $=0.2 \%$.
Therefore, $B B$ Theta ${ }^{2}=$ True. Similarly, D is the extreme point of the $4^{\text {th }}$ trend of the DC summary of threshold STheta $=0.1 \%$. However, point D is not an extreme point of a DC event of threshold $B$ Theta $=0.2 \%$. Hence, $B$ BThet ${ }^{4}=$ False. Given two distinct DC summaries, of the same price series, corresponding to two different threshold STheta and BTheta, we compute $B B$ Theta $^{i}$ for each DC event of threshold STheta. We denote by BBTheta the set of all BBTheta ${ }^{i}$.

\section{Formulation of the Forecasting Problem}

In this paper, our task is to forecast the value of BBTheta at the DCC point of a DC event of threshold STheta. For example, in TABLE I, we recognize two uptrends DC events: 1) [BB $\left.{ }^{0.1}\right]$ of threshold $0.1 \%$ and 2 ) $\left[\mathrm{BB}^{0.2}\right]$ of threshold $0.2 \% . \mathrm{B}^{0.1}$ is the DCC point of an uptrend DC event of threshold STheta $=0.1 \%$ and $\mathrm{B}^{0.2}$ is the DCC point of an uptrend DC event of threshold BTheta $=0.2 \%$. We also note two facts: 1 ) both DC events, $\left[\mathrm{BB}^{0.1}\right]$ and $\left[\mathrm{BB}^{0.2}\right]$, start at the same point $\mathrm{B}$, and 2) point $\mathrm{B}^{0.1}$ (which is observed at time 21:05:00) occurred before we observe point $\mathrm{B}^{0.2}$ (at time $\left.21: 10: 00\right)$. Note that only at point $\mathrm{B}^{0.1}$ we can confirm that point $B$ is the extreme point of an uptrend DC event of threshold STheta $=0.1 \%$.

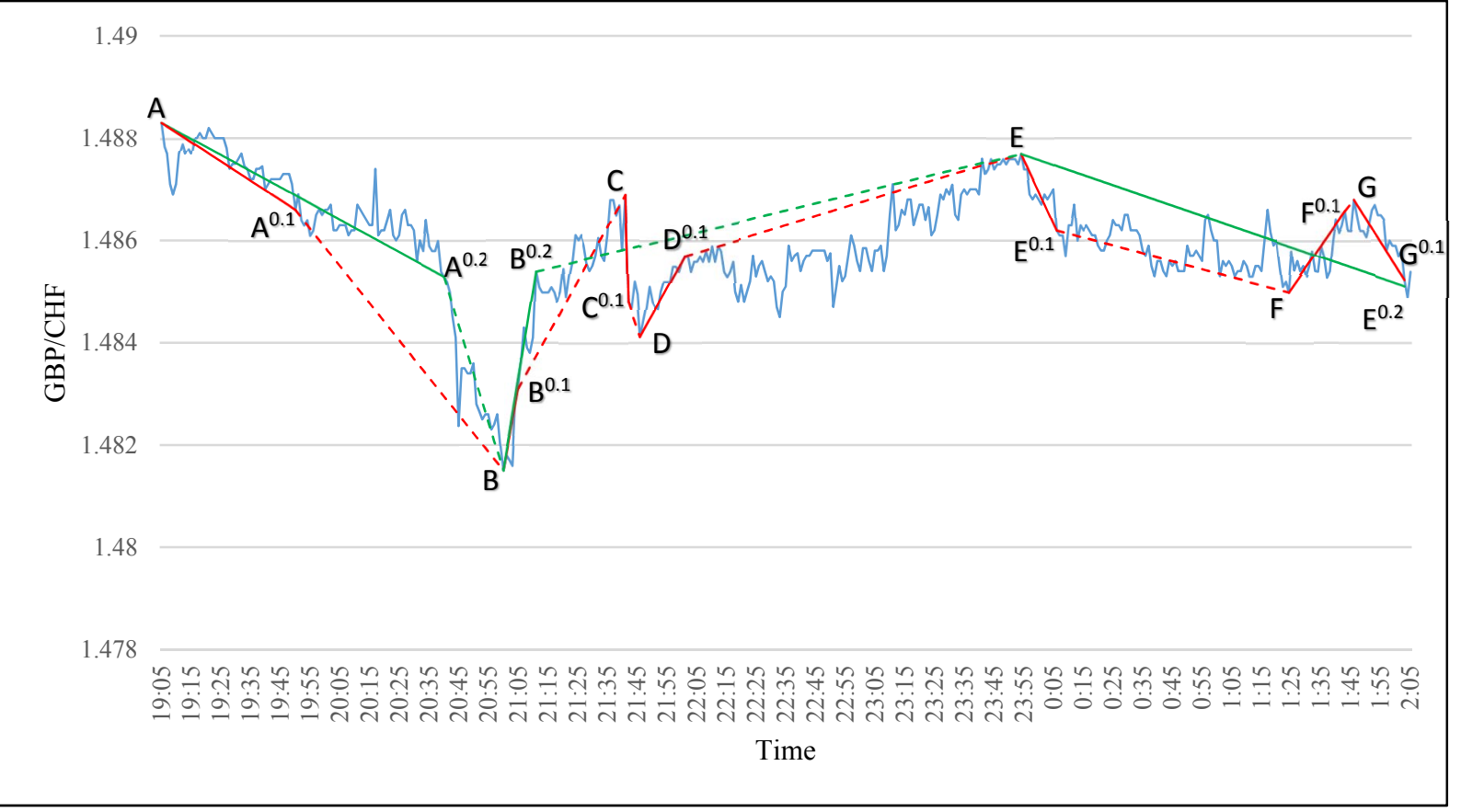

Fig. 3. The synchronization of the two DC summaries shown in Fig.1 and Fig. 2.

TABLE I. THE SYNCHRONIZATION OF THE TWO DC EVENTS [BB $\left.{ }^{0.1}\right]$ AND [BB $\left.{ }^{0.2}\right]$ SHOWN IN Fig. 3.

\begin{tabular}{|l|l|l|l|l|}
\hline Time & Mid-price & DC Summary (STheta $=\mathbf{0 . 1} \%)$ & DC Summary (BTheta $=\mathbf{0 . 2 \%})$ & Point \\
\hline $21: 00: 00$ & 1.48150 & start DC event (UPTREND) & start DC event (UPTREND) & B \\
\hline $21: 01: 00$ & 1.48180 & & & \\
\hline $21: 02: 00$ & 1.48170 & & & \\
\hline $21: 03: 00$ & 1.48159 & & & \\
\hline $21: 04: 00$ & 1.48280 & & & $B^{0.1}$ \\
\hline $21: 05: 00$ & 1.48310 & start OS event (UPTREND) & & \\
\hline $21: 06: 00$ & 1.48365 & & & \\
\hline $21: 07: 00$ & 1.48430 & & & \\
\hline $21: 08: 00$ & 1.48390 & & start OS event (UPTREND) & $B^{0.2}$ \\
\hline $21: 09: 00$ & 1.48380 & & & \\
\hline $21: 10: 00$ & 1.48540 & &
\end{tabular}


TABLE II. LIST OF NOTATIONS USED IN THIS PAPER. [11]

\begin{tabular}{|c|c|}
\hline Name / Description & Notation \\
\hline Threshold & theta \\
\hline Current price & $P_{c}$ \\
\hline Price at extreme point: price at which one trend ends and a new trend starts (e.g. points B, C in Fig. 1) & $P_{E X T}$ \\
\hline $\begin{array}{l}\text { The price, during an uptrend's OS event, required to confirm that the market's direction has changed } \\
\text { to downtrend (i.e. to confirm a downtrend's DC event). }\end{array}$ & $P_{D C C^{*}}=P_{E X T} \times(1-$ theta $)$ \\
\hline Overshoot value (OSV) at any time point. & $O S V=\left(\left(P_{c}-P_{D C C^{*}}\right) / P_{D C C^{*}}\right) /$ theta \\
\hline
\end{tabular}

However, at point $\mathrm{B}^{0.1}$ we cannot confirm whether point $\mathrm{B}$ is also an extreme point of another uptrend DC event of threshold $B$ Theta $=0.2 \%$. Only at point $\mathrm{B}^{0.2}$ we can confirm that point $\mathrm{B}$ is an extreme point of DC event of threshold BTheta and, consequently, that BBTheta ${ }^{2}$ is True. The objective, in this case, is to predict at point $\mathrm{B}^{0.1}$ whether BBTheta ${ }^{2}$ is True. In general, for the $i^{\text {th }}$ DC event of threshold STheta we want to predict whether the corresponding BBTheta ${ }^{i}$ is True. Predicting $B B$ Theta $^{i}$ to be True means that we expect that the magnitude of the $i^{\text {th }}$ trend will reach BTheta before the trend reverses.

\section{OUR APproach To ForeCASt THE END OF TREND USING BIG-THETA}

In this section we propose an approach to solve the forecasting problem described in Section III (C). The main contribution in this paper is introducing one variable to answer the question of whether BBTheta ${ }^{i}$ is True.

\section{A. The Independent Variable}

Generally, the accuracy of a forecasting model depends on the used independent variable(s). In this section we introduce one single independent variable to forecast BBTheta ${ }^{i}$ of the $i^{\text {th }}$ trend of the DC summary of threshold STheta. This variable is $O S V\left(E P_{i}^{\text {STheta }}\right.$, BTheta $)$; where $E P_{i}^{\text {STheta }}$ refer to the $i^{\text {th }}$ extreme point of the DC summary of threshold STheta. For example, let $E P_{i}^{0.001}$ denotes the $i^{\text {th }}$ element of $E P^{0.001}$. Hence, $E P_{2}^{0.001}$ and $E P_{5}^{0.001}$ represent points $\mathrm{B}$ and $\mathrm{E}$ respectively. Following, we provide an example of how to compute $\operatorname{OSV}\left(E P_{i}^{\text {STheta }}\right.$, BTheta $)$ before stating its general formula.

In Fig. 3, $\left[\mathrm{BB}^{0.1}\right]$ represents the second $\mathrm{DC}$ event of threshold STheta. Therefore, the objective is to predict whether BBTheta $^{2}$ is True. The extreme point of $\left[\mathrm{BB}^{0.1}\right]$ is $\mathrm{B}$. $\mathrm{B}$ is denoted as $E P_{2}^{0.001}$. In this case, we should compute $\operatorname{OSV}\left(E P_{2}^{0.001}, 0.002\right)$ as follow:

$$
\begin{aligned}
& \operatorname{OSV}\left(E P_{2}^{0.001}, 0.002\right)= \\
& \left(\left(P_{B}-P D C C^{* 0.002}\right) / P D C C^{* 0.002}\right) / 0.002
\end{aligned}
$$

where $P_{B}$ is the price at point B (i.e. 1.48310 from TABLE I). $P D C C^{* 0.002}$ is the $P_{D C C^{*}}$, from TABLE II, computed with respect to the last confirmed DC event of threshold BTheta; which is, in this case, $\left[\mathrm{AA}^{0.2}\right]$. Hence $P D C C^{* 0.002}=P_{A} \times(1-$ $0.002)$; where $P_{A}$ is the price at point $\mathrm{A}$.

In general, we define $\operatorname{OSV}\left(E P_{i}^{\text {STheta }}\right.$, BTheta $)$ as the overshoot value at the extreme point of the $i^{\text {th }}$ trend with respect to the DC summary of threshold BTheta. That is:

$$
\begin{aligned}
& \text { OSV }\left(E P_{i}^{\text {STheta }}, \text { BTheta }\right)= \\
& \quad\left(\left(P_{i}^{\text {STheta }}-\text { PDCC } C^{* \text { BTheta }}\right) / \text { PDCC } C^{* \text { BTheta }}\right) / \text { BTheta }
\end{aligned}
$$

Where: $P_{i}^{\text {STheta }}$ is the price at the extreme point of $i^{\text {th }} \mathrm{DC}$ event of threshold STheta. PDCC ${ }^{* B T h e t a}$ is the $P_{D C C^{*}}$ of the last confirmed DC event of threshold BTheta.

For more clarification, we provide a second example of how to compute $\operatorname{OSV}\left(E P_{i}^{S T h e t a}\right.$, BTheta $)$ based on Fig. 3. The extreme point of the uptrend $\mathrm{DC}$ event $\left[\mathrm{EE}^{0.1}\right]$ is $\mathrm{E}$. $\mathrm{E}$ is the $5^{\text {th }}$ element of $E P^{0.001}$. Hence, $\mathrm{E}$ is denoted as $E P_{5}^{0.001}$. Therefore, the objective is to predict whether BBTheta ${ }^{5}$ is True. In this case, we should compute $O S V\left(E P_{5}^{0.001}, 0.002\right)$ as in (4):

$$
\begin{aligned}
& \operatorname{OSV}\left(E P_{5}^{0.001}, 0.002\right)= \\
& \left(\left(P_{E}-P D C C^{* 0.002}\right) / P D C C^{* 0.002}\right) / 0.002
\end{aligned}
$$

Where $P_{E}$ is the price at point E. $P D C C^{* 0.002}$ is the $P_{D C C^{*}}$ computed with respect to the last confirmed DC event of threshold BTheta; which is, in this case, $\left[\mathrm{BB}^{0.2}\right]$. Note that $\left[\mathrm{BB}^{0.2}\right]$ is an uptrend DC event. Hence $P D C C^{* 0.002}=P_{B} \times(1+$ $0.002)$; where $P_{B}$ is the price at point $\mathrm{B}$.

\section{B. The Decision Tree Procedure J48}

In this paper we use the decision tree procedure, named J48, to find the relation between the two variables $B B$ Theta ${ }^{i}$ and $\operatorname{OSV}\left(E P_{i}^{\text {STheta }}\right.$, BTheta $) . \quad \mathrm{J} 48$ is the open-source Java implementation of the C4.5 algorithm [18]. J48 has three main steps. First, for each attribute $\lambda$ it computes the normalized information gain ratio from splitting on $\lambda$. Let $\lambda$ best be the attribute with the highest normalized information gain. Second, it creates a decision node $n d$ that splits on $\lambda$ best. Third, it recurs on the sub-lists obtained by splitting on $\lambda_{-}$best , and adds those nodes as children of node $n d$. The three steps are repeated until a base case is reached.

\section{Measuring the True-False Imbalance}

In Section III (B) we introduced BBTheta as the Boolean dependent variable to be predicted. Some studies (e.g. [18]) report that the performance of many machine learning algorithm can be affected by the True-False imbalance in the dependent variable.

Therefore, in this section, we introduce a variable named $\alpha$. The objective of $\alpha$ is to measure the levels of True-False imbalance in the dependent variable BBTheta. $\alpha$ is measured as the fraction of True instances of BBTheta. Let nbTrends_BTheta be the number of all trends obtained by 
running the DC summary with threshold BTheta on a particular currency pair. Similarly, let nbTrends_STheta be the number of all trends obtained by DC summary with threshold STheta. As stated in Section III, each downtrend of threshold BTheta comprises at least one downtrend of threshold STheta (the argument in case of an uptrend is similar). Thus, the fraction of extreme points of DC summary of threshold STheta those are also extreme points of DC summary of threshold BTheta is:

$$
\begin{gathered}
\alpha=\frac{\text { nbTrends_BTheta }}{\text { nbTrends_STheta }} \\
\text { V. EXPERIMENTS }
\end{gathered}
$$

Firstly, we should note that many time series forecasting techniques (e.g. ARIMA) are not applicable under the DC framework. This is because they assume that the data are sampled at regular time interval; which is not true in the case of $B B$ Theta as explained in Section III (B). In this paper, we test our forecasting approach in the FX market using three currency pairs: GBP/CHF, EUR/CHF and USD/JPY. In this section we provide two sets of experiments: 1) the objective of the first set is to evaluate the accuracy of our approach, 2) the second experiment aims to evaluate the impact of the value of BTheta on the accuracy of our forecasting approach.

\section{A. Experiment 1: Evaluating the Accuracy of the Proposed Forecasting Approach}

The objective of this experiment is to evaluate the accuracy of our forecasting approach. We apply our forecasting approach to three currency pairs; namely EUR/CHF, GBP/CHF, and USD/JPY. Each currency pair is sampled minute-by-minute from $1 / 1 / 2013$ to $31 / 7 / 2015$. As for the value of STheta and BTheta, we set different values, chosen arbitrarily, for each considered currency pair.

In preliminary experiments, we found that it would be better to forecast uptrends and downtrends, of threshold STheta, separately. Therefore, we save the uptrends and downtrends independently as two different datasets. Then, we divide each of these datasets, downtrends and uptrends, into training and out-of-sample testing periods. We use the training periods to learn the J48 decision tree. Then we use the obtained tree to make the forecast over the testing periods. The training periods range from 24 months (case of USD/JPY) to 30 months (case of EUR/CHF). The out-of-sample testing periods range from 1 month (case of EUR/CHF) to 7 months (case of USD/JPY). This diversification of training periods and out-of-sample testing periods examines the usefulness of our approach under different settings. Besides, we compare the accuracy of our approach with ARIMA forecasting technique.

\section{B. Experiment 2: The Impact of BTheta on the Accuracy of Forecasting}

In this experiment we aim to check whether the accuracy of our approach can be affected by the value of BTheta. To this end, for each of the three considered currency pairs, we apply our forecasting approach using ten different values of BTheta. We measure the ten obtained accuracies. In this experiment, STheta is set to $0.1 \%$. For each currency pair, the training and testing periods are sets to be the same as in Experiment 1.
Note that the value of $\alpha$, as in (5), depends on the value of BTheta. Consequently, by choosing ten different values of BTheta, we obtain ten different levels of True-False imbalance in the dependent variable BBTheta. Thus, we can use the results of this experiment to measure the accuracy of our approach under different levels of True-False imbalance.

\section{REsults AND Discussion}

\section{A. Experiment 1: Evaluating the Accuracy of the Proposed Forecasting Approach}

The objective of this experiment is to evaluate the performance of our forecasting approach in the FX market. To this end, we apply it to three currency pairs, namely EUR/CHF, GBP/CHF, and USD/JPY. For each currency pair we consider the uptrends and downtrends, of the DC summary of threshold STheta, separately. We also try different values of thresholds STheta and BTheta, chosen arbitrarily. The experimental results and parameters' values are reported in TABLE III. In TABLE III, the first column to the left specifies the considered currency pair. The second and third columns denote the values of STheta and BTheta respectively. The column ' $\alpha$ ' denotes the TrueFalse unbalance corresponding to the specified currency pair. The column 'Type of Trend' specifies whether the set of uptrends or downtrends, corresponding to DC analysis of STheta, is considered. The column 'Accuracy' show the accuracy obtained by applying our approach to the testing period. The accuracy is computed as:

$$
\text { Accuracy }=\frac{T P+T N}{N}
$$

Where $N$ is the number of instances of BBTheta. TP is the number of correctly forecasted True instances of BBTheta. TN is the number of correctly forecasted False instances of BBTheta. The columns 'Trading Period' and 'Out-of-sample Testing Period' indicate the length of training periods and testing period, respectively, for each currency pair. As can be seen in TABLE III, for different training and testing periods and for the different selected values of STheta and BTheta, all obtained accuracies are above $80 \%$. These results show that the proposed independent variable, in (3), is very useful to forecast DC.

We compare the accuracy of our approach with ARIMA forecasting technique. For this purpose, we use the function auto.arima() from the package 'forecast' of the statistical software R to predict BBTheta ${ }^{i}$. The accuracy of using ARIMA is reported in last column to the right in TABLE III. As can be seen the accuracy of our approach outperforms ARIMA in all cases.

\section{B. Experiment 2: The Impact of BTheta on the Accuracy of Forecasting}

The objective of this experiment is to examine whether the value of BTheta may affect the accuracy of the forecasting approach proposed in this paper. To this end, we apply our forecasting approach to each of the considered three currency pairs using ten different values of BTheta. 
TABLE III. THE SETTINGS AND RESULTS OF EXPERIMENT 1. THE REPORTED ACCURACIES CORRESPSOND TO THE OUT-OF-SAMPLE TESTING PERIODS.

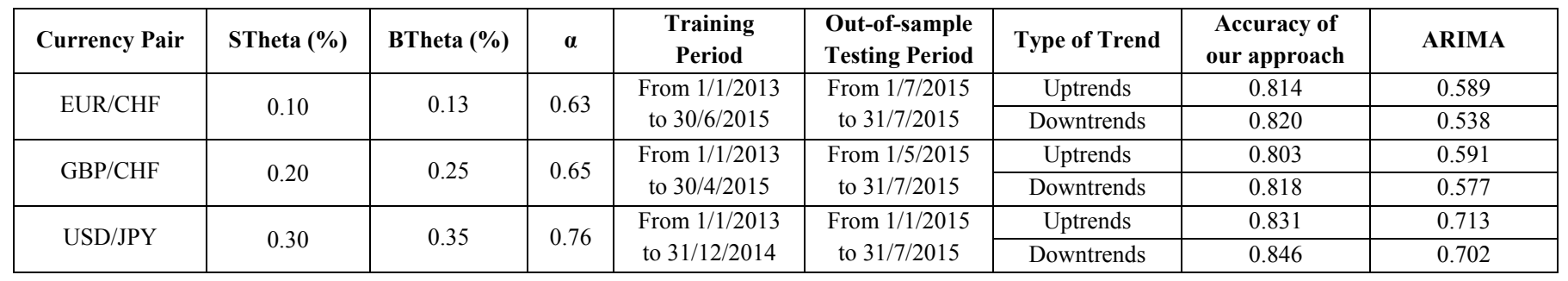

TABLE IV. ANALYZING THE IMPACT OF VALUE OF BTHETA ON THE ACCURACY OF OUR FORECASTING APPROACH. STHETA IS FIXED TO $0.10 \%$. ALL REPORTED RESUTLS CORRESPOND TO THE OUT-OF-SAMPLE TESTING PERIODS.

\begin{tabular}{|c|c|c|c|c|c|c|c|}
\hline \multirow{3}{*}{ Type of Trend } & \multirow{2}{*}{ BTheta (\%) } & \multicolumn{2}{|c|}{ EUR/CHF } & \multicolumn{2}{c|}{ GBP/CHF } & \multicolumn{2}{c|}{ USD/JPY } \\
\cline { 2 - 8 } & & Accuracy & $\boldsymbol{\alpha}$ & Accuracy & $\boldsymbol{\alpha}$ & Accuracy & $\boldsymbol{\alpha}$ \\
\hline \multirow{7}{*}{ Uptrends } & 0.13 & 0.815 & 0.63 & 0.818 & 0.64 & 0.822 & 0.64 \\
\cline { 2 - 8 } & 0.14 & 0.780 & 0.54 & 0.789 & 0.55 & 0.796 & 0.56 \\
\cline { 2 - 8 } & 0.15 & 0.744 & 0.48 & 0.754 & 0.49 & 0.766 & 0.50 \\
\cline { 2 - 8 } & 0.16 & 0.718 & 0.42 & 0.733 & 0.42 & 0.737 & 0.45 \\
\cline { 2 - 8 } & 0.17 & 0.698 & 0.37 & 0.708 & 0.37 & 0.714 & 0.40 \\
\cline { 2 - 8 } & 0.18 & 0.672 & 0.33 & 0.688 & 0.33 & 0.695 & 0.36 \\
\cline { 2 - 8 } & 0.19 & 0.654 & 0.30 & 0.672 & 0.30 & 0.681 & 0.33 \\
\cline { 2 - 8 } & 0.20 & 0.642 & 0.27 & 0.637 & 0.27 & 0.637 & 0.30 \\
\cline { 2 - 8 } & 0.21 & 0.632 & 0.25 & 0.636 & 0.25 & 0.649 & 0.28 \\
\hline & 0.22 & 0.619 & 0.22 & 0.621 & 0.23 & 0.639 & 0.26 \\
\hline & 0.13 & 0.820 & 0.63 & 0.810 & 0.64 & 0.803 & 0.64 \\
\cline { 2 - 8 } & 0.14 & 0.779 & 0.54 & 0.774 & 0.55 & 0.770 & 0.56 \\
\cline { 2 - 8 } & 0.15 & 0.747 & 0.48 & 0.748 & 0.49 & 0.739 & 0.50 \\
\cline { 2 - 8 } & 0.16 & 0.720 & 0.42 & 0.709 & 0.42 & 0.715 & 0.45 \\
\cline { 2 - 8 } & 0.17 & 0.693 & 0.37 & 0.697 & 0.37 & 0.698 & 0.40 \\
\cline { 2 - 8 } & 0.18 & 0.670 & 0.33 & 67.8 & 0.33 & 0.672 & 0.36 \\
\cline { 2 - 8 } & 0.19 & 0.655 & 0.30 & 0.661 & 0.30 & 0.649 & 0.33 \\
\cline { 2 - 7 } & 0.20 & 0.642 & 0.27 & 0.639 & 0.27 & 0.657 & 0.30 \\
\cline { 2 - 7 } & 0.21 & 0.635 & 0.25 & 0.635 & 0.25 & 0.629 & 0.28 \\
\cline { 2 - 7 } & 0.22 & 0.620 & 0.22 & 0.627 & 0.23 & 0.620 & 0.26 \\
\hline
\end{tabular}

The results of out-of-sample testing of this experiment are reported in TABLE IV. The training and out-of-sample testing periods of each currency pairs are the same adopted in TABLE III. In each of the three columns ('EUR/CHF', 'GBP/CHF', and 'USD/JPY') we note that the accuracy of our approach increases as BTheta decreases. To validate this note statically, we apply linear regression with the dependent variable being the column 'BTheta (\%)' and the independent variable being the column 'Accuracy' for each of the three currency pairs' column. The obtained p-value corresponding to 'BTheta (\%)' is less than 0.01 for each of these currency pairs. This is less than the common level of 0.05 ; which indicates that BTheta is statistically significant for the computation of the accuracy. Logically, if BTheta becomes equal to STheta then $\alpha=1$. In this case, however, there is no need for learning to be done and the considered forecasting problem becomes meaningless.

Furthermore, the results of Experiment 2 allow us to evaluate the performance of our forecasting approach under different levels of True-False imbalance (i.e. $\alpha$ ). For example, in the TABLE IV, in column 'EUR/CHF', we note that $\alpha$ ranges between 0.22 (i.e. $22 \%$ of BBTheta instances are True) and 0.63 (i.e. $63 \%$ of BBTheta instances are True). The corresponding accuracies range between 0.627 and 0.818 . As for the results corresponding to the column ' $\mathrm{GBP} / \mathrm{CHF}$ ', we note that $\alpha$ ranges between 0.26 and 0.64 . The corresponding accuracies range between 0.639 and 0.822 . Based on the results reported in the column 'USD/JPY' the range of $\alpha$ is between 0.23 and 0.64 . The range of accuracy is between 0.620 and 0.822 .

These results highlight two advantages: 1) the accuracy of our approach is quite good for many levels of True-False imbalance in the dependent variable BBTheta, and 2) the accuracy of our approach is reasonably consistent among the three considered currency pairs.

However, these results also highlights two limitations: 1) In general, the accuracy of our forecasting model decreases as the difference between BTheta and STheta increases; and 2) When the difference between BTheta and STheta becomes greater than a specific value, our model cannot outperform dummy prediction. For example, in the column of 'EUR/CHF' we note that for BTheta greater than $0.19 \%$ (case of 'uptrends') the corresponding $\alpha$ becomes less than 0.30 . In such case, the accuracy of dummy prediction (which predict always False) is expected to be 0.70 . Whereas, the forecasting accuracy of our approach is less than 0.65 . 


\section{CONCLUSION}

The FX market is the most liquid financial market. Due to its extreme importance for macro- and microeconomic decision making, many approaches have been developed to forecast exchange rate and trend's direction in the FX market. Most of existing studies, those address the problem of trend's direction prediction, use data sampled at regular time interval; which is commonly known as time series.

The Directional Changes (DC) framework is an approach to sample prices in financial market other than time series. In the context of DC, a trend is defined as a price change greater than, or equal to, a specific threshold theta. If the price rises by theta then the market is in uptrend. An uptrend ends whenever the price drops by theta (in this case we say that the market switches to downtrend) and vice versa. In the DC context, the uptrends and downtrends alternate. The DC approach for sampling prices movement has been proved many times to be useful in analyzing the FX market.

In this paper we study the problem of trend's direction prediction under the DC framework in the FX market. This problem has not been proposed in the previous DC studies. Our task is to forecast how far, in terms of price change, the current trend will continue before the trend changes.

The main contribution of this paper is identifying only one independent variable and proving that it is relevant to the introduced prediction problem. The experimental results are conducted using three currency pairs: EUR/CHF, GBP/CHF, and USD/JPY. The experimental results suggest that the accuracy of our approach is reasonably consistent among the considered currency pairs. In some cases, the accuracy was over $80 \%$. This prove that DC are predictable and that the introduced independent variable is helpful to forecast the end of a trend under the DC framework. However, our forecasting approach was outperformed by dummy prediction for some specific levels of True-False unbalance of dependent variable.

Having established the predictive power of our approach, our next goal will be to embed this forecasting result into trading strategies.

\section{REFERENCES}

[1] Monitory and Economic Department, "Triennial Central Bank Survey Foreign exchange turnover in April 2016," Bank for International Settlements, 2016.

[2] L. Maciel, F. Gomide, D. Santos and R. Ballini, "Exchange rate forecasting using echo state networks for trading strategies," in IEEE Conference on Computational Intelligence for Financial Engineering \& Economics (CIFEr), London, 2014.
[3] F. Bahramy and S. F. Crone, "Forecasting foreign exchange rates using Support Vector Regression," in IEEE Conference on Computational Intelligence for Financial Engineering \& Economics (CIFEr), Singapore, 2013.

[4] L. Szu-Yin, C. Chi-Hua and L. Chi-Chun, "Currency Exchange Rates Prediction based on Linear Regression Analysis Using Cloud Computing," International Journal of Grid and Distributed Computing, vol. 6, no. 2, pp. 1-9, 2013.

[5] V. Pacelli, "Forecasting Exchange Rates: a Comparative Analysis," International Journal of Business and Social Science, vol. 3, no. 10, pp. 145-156, 2012.

[6] L. M. Badea, "Forecasting Exchange Rates with Mixed Models," Journal of Mobile, Embedded and Distributed Systems, vol. 5, no. 2, pp. 84-89, 2013.

[7] S.H. Park, J.H. Lee, J.W. Song and T.S. Park, "Forecasting Change Directions for Financial Time Series Using Hidden Markov Model," in Rough Sets and Knowledge Technology, vol. 5589, Springer Berlin Heidelberg, 2009, pp. 184-191.

[8] A. Skabar, "Direction-of-Change Financial Time Series Forecasting using Bayesian Learning for MLPs," in Proceedings of the World Congress on Engineering, London, UK., 2008.

[9] A. Azzini, C. d. C. Pereira and A. G. B. Tettamanzi, "Predicting Turning Points in Financial Markets with Fuzzy-Evolutionary and NeuroEvolutionary Modeling," in Applications of Evolutionary Computing, vol. LNCS 5484, M. Giaboni, et al., Eds., Springer, 2009, pp. 213-222.

[10] E. Tsang, "Directional Changes, Definition," Working Paper WP050-10, Centre for Computational Finance and Economic Agents (CCFEA), University of Essex, Colchester, 2010.

[11] E. P. K. Tsang, R. Tao and S. Ma, "Profiling Financial Market Dynamics under Directional Changes," Quantitative finance, http://www.tandfonline.com/doi/abs/10.1080/14697688.2016.1164887, published online on 07 Jun 2016.

[12] J. Glattfelder, A. Dupuis and R. Olsen, "Patterns in high-frequency FX data: Discovery of 12 empirical scaling laws," Quantitative Finance, vol. 11, no. 4, pp. 599-614, 2011.

[13] T. Bisig, A. Dupuis, V. Impagliazzo and R. Olsen, "The scale of market quake," Quantitative Finance, vol. 12, no. 4, pp. 501-508, 2012.

[14] A. Dupuis and R. Olsen, "High Frequency Finance: Using Scaling Laws to Build Trading Models," in Handbook of Exchange Rates, J. James et al, Ed., NJ,USA, Wiley, 2012, pp. 563-582.

[15] S. Masry, "Event-Based Microscopic Analysis of the FX Market," PhD thesis, University of Essex, Colchester, 2013.

[16] J. Gypteau, F. Otero and M. Kampouridis, "Directional Changes based trading strategy with genetci programming," in EvoStar, EvoApplication, Mora, A. M. and Squillero, G. Eds., Denmark, Springer, 2015, pp. 1-12.

[17] A. Golub, G. Chliamovitch A. Dupuis and B. Chopard, "Multi-Scale Representation of High Frequency Market Liquidity," SSRN, 2014. [Online]. http://papers.ssrn.com/sol3/papers.cfm?abstract_id=2393428.

[18] J. Quinlan, C4.5: Programs for Machine Learning, San Francisco, CA,USA: Morgan Kaufmann Publishers Inc., 1993. 